\title{
Focus on neuro-critical care: combined interventions to improve relevant outcomes
}

\author{
Chiara Robba', Romain Sonneville ${ }^{2,3}$ and Geert Meyfroidtt, , $^{*}$
}

(c) 2020 Springer-Verlag GmbH Germany, part of Springer Nature

Management of patients suffering from acute neurological injury, such as traumatic brain injury (TBI) or stroke, mandates high-quality care throughout the whole trajectory, including adequate triage for multi-casualty trauma [1], care path bundles, and early revascularization for stroke [2], antifibrinolytic agents in specific subgroups of TBI patients [3], and prevention as well as treatment of associated systemic effects, such as electrolyte disturbances or alterations in kidney function [4]. The benefits and safety of early mobilization in the intensive care unit (ICU) are well established. However, in a sub-study of 200 patients from the Surgical ICU Optimal Mobilization Score (SOMS) trial [5], patients with an admission Glasgow Coma Scale (GCS) $<8$ tended to be mobilized later, even while the benefits of mobilization were also present in this subgroup with severe neurological impairment. The management of respiratory failure can be challenging in patients with acute brain injury, especially when there is concern about increased intracranial pressure (ICP) or secondary brain injury. Nevertheless, the literature on lung-protective ventilation in these patients suggests that low tidal volume, high PEEP, and even rescue therapies, such as prone ventilation, could be applied here, with caution and close monitoring [6]. Neuroprotection is mainly driven by balancing substrate and oxygen delivery and demand to the brain [7]. Alternative energy substrates, such as lactate, or ketone bodies, may be potential future options to boost cerebral energy metabolism [8], but further prospective trials are needed to demonstrate their theoretical benefits. Impaired cerebral blood flow $(\mathrm{CBF})$ plays an important role in secondary brain injury.

\footnotetext{
*Correspondence: geert.meyfroidt@uzleuven.be

${ }^{4}$ Department of Intensive Care Medicine, University Hospitals Leuven, Leuven, Belgium
}

Full author information is available at the end of the article
Brain ultrasonography is a noninvasive method that allows to assess structural brain abnormalities, as well as a valid option to assess CBF-changes [9]. This technique can be taught easily and is increasingly being used in the ICU and the emergency department. The large prospective observational CENTER-TBI study [10] has demonstrated striking regional and national variations in TBI management across different regions and countries. There is wide agreement amongst experts, supported by experimental work, cohort studies, and clinical expertise that a neuromonitoring-based treatment approach allows for a rational and physiology-based management in TBI, even while evidence from randomized-controlled trials for improved clinical outcomes through such strategy is currently lacking. The recently published Seattle International Severe Traumatic Brain Injury Consensus Conference (SIBICC) guidelines now provide algorithms for ICP-based adult TBI management [11]. Using a Delphimethod-based approach, experts recommend 18 interventions as fundamental and 10 treatments not to be used for the treatment of elevated ICP. A comprehensive practical tiered management algorithm was designed, to aid clinicians in their choice on the appropriate therapy.

In spite of the evolutions in management, a significant proportion of survivors from a severe acute brain injury will suffer from long-lasting or even permanent disabilities that potentially interfere with their quality of life. The burden of TBI on individuals and society remains huge: in CENTER-TBI [10], TBI mortality was lower than expected, but more than half of the moderate to severe TBI patients had unfavorable outcomes at 6 months. Similarly, while the mortality after Herpes Simplex encephalitis has significantly decreased over the past years, a retrospective multicenter study found that $29-43 \%$ of survivors continued to suffer from significant long-term disabilities [12]: 90 days after ICU admission, $71 \%$ had a modified Rankin Score (mRS) of 3-6, which

\section{Springer}




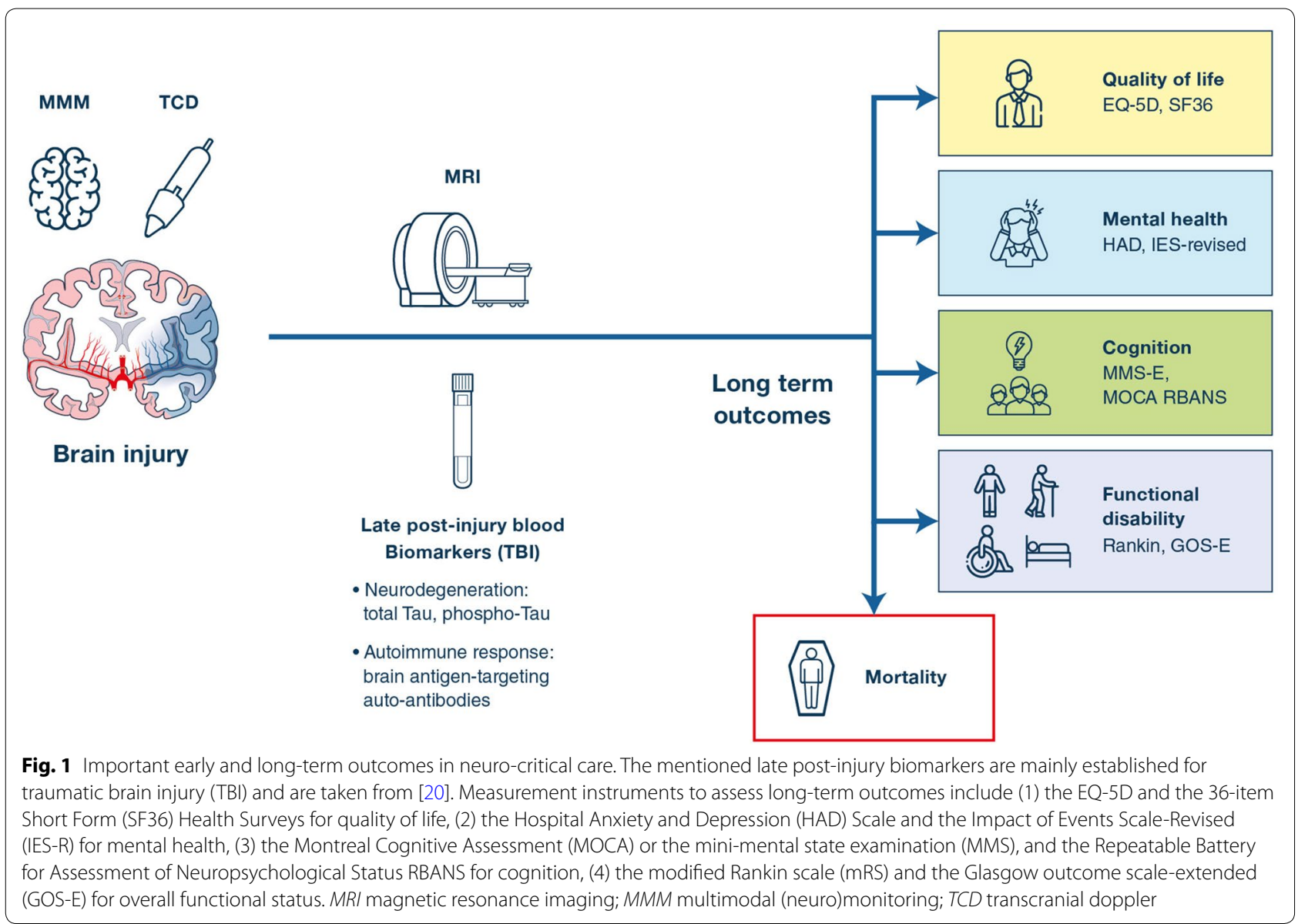

corresponds with a wide range of disabilities, from mild motoric problems (but able to walk independently), to being bedridden, or death. In contrast, non-infectious auto-immune encephalitis, when recognized in time, and with appropriate aggressive therapy, in general has a good outcome, even when the ICU length of stay is prolonged [13]. Focusing only on survival of critically ill patients without considering their long-term quality of life and cognitive psychological and physical impairments is no longer acceptable. The mRS and the Glasgow Outcome Scale (GOS) or GOS-Extended (GOS-E) have been the most frequently used outcome scales in neuro-critical care research. For statistical convenience in research studies, they are often dichotomized into good versus bad outcome, which may be acceptable when an intervention has a uniform benefit over a wide range of injury severity [14]. However, these scales, certainly when dichotomized, may obscure a wide spectrum of subtle or even overt disabilities that will matter to the quality of life of patients, or their relatives. Chronic progression of brain injury can persist and evolve over years, through several mechanisms, including chronic inflammation, loss or gains of connectivity, or brain atrophy. Longitudinal functional MRI studies [15] have demonstrated white matter changes associated with late recovery. Loss of brain volume and atrophy can be sensitively measured using volumetric analysis of MRI [16]. Figure 1 is a summary of potential clinical or mechanistic outcome measures for brain injury. Outcome assessment is usually performed relatively early after the injury (for instance at 28 or 90 days). The initial speed of neurological recovery can be informative on longer-term outcomes, as was done in the amantadine trial for prolonged vegetative or minimally conscious states after TBI [17]. Complete rehabilitation from a severe neurological injury may take months or longer. For instance, in the large randomizedcontrolled RESCUE-ICP on decompressive craniectomy as a last tier therapy [18], the benefits of the intervention on GOS-E only became apparent at 12-month follow-up, indicating a significant progression from 6 to 12 months. In that perspective, relatively short follow-up intervals to assess outcomes are not always appropriate. Long-term follow-up, however, is hard to achieve, and at least 15\% loss to follow-up at 1 year is to be expected [19].

Even while novel agents or therapies with a demonstrated effect on outcomes in brain injured patients 
have not been discovered recently, longitudinal epidemiological studies do demonstrate that over decades, and intensive care medicine has been successful in gradually reducing the mortality. Future studies should focus on longer-term patient centered functional outcomes and quality of life, even while collecting these data is a huge effort.

\section{Author details}

${ }^{1}$ Anesthesia and Intensive Care, Policlinico San Martino, IRCCS for Oncology and Neuroscience, Genoa, Italy. ${ }^{2}$ INSERM UMR1 148, Team 6, Université de Paris, 75018 Paris, France. ${ }^{3}$ Department of Intensive Care Medicine and Infectious Diseases, AP-HP, Bichat-Claude Bernard Hospital, 75018 Paris, France. ${ }^{4}$ Department of Intensive Care Medicine, University Hospitals Leuven, Leuven, Belgium. ${ }^{5}$ Laboratory of Intensive Care Medicine, University of Leuven (KU Leuven), Leuven, Belgium.

\section{Funding}

None.

\section{Compliance with ethical standards}

\section{Conflicts of interest}

GM is associate Editor of ICM. GM is funded by the Research Foundation Flanders (Fonds Wetenschappelijk Onderzoek) as senior clinical investigator and receives project funding (C2) from the KULeuven, Belgium. CR is Junior Editor of ICM. RS received grants from the French Ministry of Health, the French society of intensive care medicine (SRLF) and the European society of intensive care medicine (ESICM), and lecture fees from Baxter.

\section{Publisher's Note}

Springer Nature remains neutral with regard to jurisdictional claims in published maps and institutional affiliations.

Received: 5 February 2020 Accepted: 16 March 2020

Published online: 23 March 2020

\section{References}

1. Raux M, Carli P, Lapostolle F et al (2019) Analysis of the medical response to November 2015 Paris terrorist attacks: resource utilization according to the cause of injury. Intensive Care Med 45:1231-1240. https://doi.org/10.1007/ s00134-019-05724-9

2. Smith M, Reddy U, Robba C et al (2019) Acute ischaemic stroke: challenges for the intensivist. Intensive Care Med 45:1177-1189. https://doi. org/10.1007/s00134-019-05705-y

3. Taccone FS, Citerio G, Stocchetti N (2019) Is tranexamic acid going to CRASH the management of traumatic brain injury? Intensive Care Med. https://doi. org/10.1007/s00134-019-05879-5

4. Legrand M, Sonneville R (2019) Understanding the renal response to brain injury. Intensive Care Med 45:1112-1115. https://doi.org/10.1007/s0013 4-019-05685-z
5. Schaller SJ, Scheffenbichler FT, Bose S et al (2019) Influence of the initial leve of consciousness on early, goal-directed mobilization: a post hoc analysis. Intensive Care Med 45:201-210. https://doi.org/10.1007/s00134-019-05528 $-x$

6. Frisvold SK, Robba C, Guérin C (2019) What respiratory targets should be recommended in patients with brain injury and respiratory failure? Intensive Care Med 45:683-686. https://doi.org/10.1007/s00134-019-05556-7

7. Stocchetti N, Taccone FS, Citerio G et al (2015) Neuroprotection in acute brain injury: an up-to-date review. Crit Care 19:186. https://doi.org/10.1186/ s13054-015-0887-8

8. Oddo M, Vespa P, Menon DK (2019) Boosting the injured brain with supplemental energy fuels. Intensive Care Med 45:872-875. https://doi. org/10.1007/s00134-018-05517-6

9. Robba C, Goffi A, Geeraerts T et al (2019) Brain ultrasonography: methodology, basic and advanced principles and clinical applications-a narrative review. Intensive Care Med 45:913-927. https://doi.org/10.1007/s0013 4-019-05610-4

10. Steyerberg EW, Wiegers E, Sewalt C et al (2019) Case-mix, care pathways, and outcomes in patients with traumatic brain injury in CENTER-TBI: a European prospective, multicentre, longitudinal, cohort study. Lancet Neurol 18:923-934. https://doi.org/10.1016/S1474-4422(19)30232-7

11. Hawryluk GWJ, Aguilera S, Buki A et al (2019) A management algorithm for patients with intracranial pressure monitoring: the Seattle International Severe Traumatic Brain Injury Consensus Conference (SIBICC). Intensive Care Med 45:1783-1794. https://doi.org/10.1007/s00134-019-05805-9

12. Jaquet $P$, De Montmollin E, Dupuis C et al (2019) Functional outcomes in adult patients with herpes simplex encephalitis admitted to the ICU: a multicenter cohort study. Intensive Care Med 45:1 103-1111. https://doi. org/10.1007/s00134-019-05684-0

13. Sonneville R, Venkatesan A (2019) Honnorat J (2019) Understanding autoimmune encephalitis in the ICU. Intensive Care Med 45(12):1795-1798

14. Saver JL, Gornbein J (2009) Treatment effects for which shift or binary analyses are advantageous in acute stroke trials. Neurology 72:1310-1315. https ://doi.org/10.1212/01.wnl.0000341308.73506.b7

15. Newcombe VFJ, Correia MM, Ledig C et al (2016) Dynamic changes in white matter abnormalities correlate with late improvement and deterioration following TBI. Neurorehabil Neural Repair 30:49-62. https://doi. org/10.1177/1545968315584004

16. Cole JH, Jolly A, De Simoni S et al (2018) Spatial patterns of progressive brain volume loss after moderate-severe traumatic brain injury. Brain 141(3):822-836

17. Giacino JT, Whyte J, Bagiella E et al (2012) Placebo-controlled trial of amantadine for severe traumatic brain injury. N Engl J Med 366:819-826. https:// doi.org/10.1056/NEJMoa1102609

18. Hutchinson PJ, Kolias AG, Timofeev IS et al (2016) Trial of decompressive craniectomy for traumatic intracranial hypertension. N Engl J Med 375:1119-1130. https://doi.org/10.1056/NEJMoa1605215

19. Richter S, Stevenson S, Newman T et al (2019) Handling of missing outcome data in traumatic brain injury research: a systematic review. J Neurotrauma 36:2743-2752. https://doi.org/10.1089/neu.2018.6216

20. Wang KK, Yang Z, ZhuT et al (2018) An update on diagnostic and prognostic biomarkers for traumatic brain injury. Expert Rev Mol Diagn 18:165-180. https://doi.org/10.1080/14737159.2018.1428089 